\title{
Temporomandibular Joint Disorders as a Cause of Aural Fullness
}

\author{
Yongxin Peng \\ Otolaryngology Department, PLA Army General Hospital, Beijing, China
}

Objectives. Temporomandibular joint disorders (TMD) are often associated with aural manifestations. However, it is not clear whether aural fullness could be induced by TMD. The purpose was to investigate the TMD and effectiveness of TMD treatments in patients with mainly or exclusively aural fullness complaint.

Methods. One hundred and twelve patients, who had aural fullness as the main or sole complaint, presented to the Otolaryngology Department, PLA Army General Hospital, Beijing, China, between January 2010 and January 2015. Patients' medical history indicated that they had previously been diagnosed and treated for otitis media or sensorineural hearing loss but without positive results. Patients were subjected to pure tone audiometry and acoustic immittance screening using GSI-61 clinical audiometer and GSI TympStar middle ear analyzer respectively. Patients were examined by questionnaire, $\mathrm{X}$-ray and/or computed tomography scan of temporomandibular joint. TMD was categorized according to the Research Diagnostic Criteria for Temporomandibular Disorders (RDC/TMD). Patients were then treated forTMD.

Results. All the patients showed normal eardrum and type A tympanogram. The patients of $60.7 \%$ (68/112) were classified as group ITMD disorders (muscle disorders), 34.8\% (39/112) were group II (disc displacements), and 4.5\% (5/112) were group III (arthralgia, osteoarthritis, and osteoarthrosis). Aural fullness was completely resolved or significantly improved in 67 and 34 patients respectively following treatments aimed at improving TMD, with a combined effectiveness of $90.2 \%$ (101/112).TMD treatments are especially effective (94.1\%) in group I TMD.

Conclusion. TMD as a potential cause of aural fullness should be considered in otolaryngology practice.

Keywords. Temporomandibular Joint Disorders; Aural Fullness; Otolaryngology

\section{INTRODUCTION}

Temporomandibular joint disorders (TMD) are a group of musculoskeletal conditions that involve the temporomandibular joints, the masticatory muscles and all associated tissues [1]. Common signs and symptoms of TMD include temporomandibular joint or masticatory muscle pain, restricted mouth opening,

- Received July 28, 2016

Revised December 17, 2016

Accepted December 27, 2016

- Corresponding author: Yongxin Peng

Otolaryngology Department, PLA Army General Hospital, Nanmencang 5,

Dongcheng District, Beijing 100010, China

Tel: +86-130-2101-6459, Fax: +86-010-8563-0233

E-mail: yy20052007@gmail.com clicking or crepitus of the temporomandibular joint, headache in the temporal region, otalgia, tinnitus, and hearing loss [2]. Numerous studies have reported a link between the aural symptoms and TMD [3-8]. However, TMD as a potential cause of otologic symptoms, in particular aural fullness, is often neglected in clinical practice by otolaryngologists. As a result, patients with aural fullness as the main or sole complaint could be misdiagnosed, leading to ineffective treatment and prolonged suffering. This study examined the presence of TMD and effectiveness of TMD treatment in 112 Chinese patients, who presented to the otolaryngology clinics for aural fullness and had been diagnosed and treated for otitis media or sensorineural hearing loss without positive results.

Copyright (c) 2017 by Korean Society of Otorhinolaryngology-Head and Neck Surgery.

This is an open-access article distributed under the terms of the Creative Commons Attribution Non-Commercial License (http://creativecommons.org/licenses/by-nc/4.0)

which permits unrestricted non-commercial use, distribution, and reproduction in any medium, provided the original work is properly cited. 


\section{MATERIALS AND METHODS}

\section{Ethics and registration}

This study was approved by the ethics committee of PLA Army General Hospital, Beijing, (No. 2016-37) and is fully compliant with the International Council for Harmonisation of Technical Requirements for Pharmaceuticals for Human Use Good Clinical Practice (ICH-GCP) and related regulation and law of China. Written informed consent was obtained from each participant or each participant's guardian before data collection.

\section{Patient cohort}

The study involved 112 patients registered for aural fullness between January 2010 and January 2015 at the Otolaryngology Department, PLA Army General Hospital, Beijing, China. The patient cohort includes 40 males and 72 females with ages ranging from 12 to 76 years old and an average of 38 years. Of the 112 patients, 94 had unilateral aural fullness and 18 had bilateral aural fullness. Seventy nine identified aural fullness as the main complaint and 33 indicated aural fullness as the sole symptom. The course of aural fullness was between 2 days to 2 months. The medical history indicated that 90 patients had been treated as otitis media using a combination of antibiotics and steroids for 7 to 14 days, of which 15 underwent tympanum puncture, and the remaining 22 patients were treated as sensorineural deafness for 7 to 25 days with regimens to improve blood supply to the cochlea and neural loss. None of the treatments was effective of improving aural fullness.

\section{Aural fullness grading}

To assess the level of aural fullness, a three-level grading system was established based on patient's perception of the symptom. The criteria are as follows. Grade 1 (mild) aural fullness is present but not affecting daily activities and patients do not actively seek therapy. Grade 2 (moderate) aural fullness is obvious but tolerable, interfering with daily activities, and patients actively seek therapy. Grade 3 (severe) aural fullness is intolerable and severely disrupting daily activities; patients are irritable, experiencing insomnia and seeking immediate therapy. Treatment is considered effective if the aural fullness grade is reduced by at

\section{H I G H L I G H T S}

- Patients with aural fullness as the sole or main complaint often have temporomandibular joint disorders (TMD).

- Treatments for TMD can resolve or significantly improve aural fullness.

- Physical therapy is the most effective against aural fullness in group I TMD patients.

- This study provides evidence that TMD may underlie aural fullness. least 1 . A reduction less by 1 or recurrence of aural fullness is indicative of ineffective treatment.

\section{TMD diagnosis}

Patients were questioned for presence of common TMD symptoms such as restricted mouth opening, temporomandibular joint pain, clicking or crepitus of the temporomandibular joint, etc. Patients were subjected to pure tone audiometry and acoustic immittance screening using GSI-61 clinical audiometer (Minnesota, MN, USA) and GSITympStar middle ear analyzer (GSI) respectively. Temporomandibular joint was further examined by $\mathrm{X}$-ray or computed tomography (CT) scan to help categorizing TMD. All of the 112 patients were diagnosed according to the Research Diagnostic Criteria for Temporomandibular Disorders (RDC/TMD) [9] and categorized into three groups: I, muscle disorders; II, disc displacements; and III, arthralgia, osteoarthritis, and osteoarthrosis.

\section{Treatments}

Based on the clinical manifestations and responsiveness to therapy, patients were treated for TMD at the Otolaryngology Department for 2 days to 4 months, using the following approaches in a sequential manner: physiotherapy (heat therapy, acupuncture and massage), intra-articular injection of glucocorticoid and sodium hyaluronate, orthodontics and occlusal equilibration. Physiotherapy was first used given its noninvasive nature, low cost, and the ease to perform. If physiotherapy failed to improve the aural fullness symptom, glucocorticoid injection was used in those showing group I disorders (muscle disorders). Sodium hyaluronate injection was used to treat patients showing joint structure abnormalities. Due the adverse effect associated with sodium hyaluronate injection, only 11 patients consented to this treatment. Orthodontics and occlusal equilibration were used to treat occlusion disorders. Finally, elastic bondage wrapping and surgery were used to treat recurrent joint dislocation or bone fracture in 3 patients. Forty-five patients voluntarily participated in follow-up surveys 10-18 months posttreatments and were examined for aural fullness.

\section{RESULTS}

\section{Correlation between aural fullness and TMD}

Major clinical manifestations of the patient cohort were listed in Table 1. Eighty-nine patients passed the pure tone audiometry (multiple frequency air-mediated hearing threshold $<25 \mathrm{~dB}$ ), and 22 patients showed unilateral or bilateral hearing loss at 4-8 $\mathrm{kHz}$ (threshold between $30-70 \mathrm{~dB}$ ). All the 112 patients included in the study exhibited air-bone gap $\leq 5 \mathrm{~dB}$ and air conduction threshold $\leq 25 \mathrm{~dB}$ in pure tone audiometry (125-1,000 $\mathrm{Hz}$ ). All patients exhibited type A tympanogram according to the Liden/Jerger classification system. X-ray scan was performed 
Table 1. Major clinical manifestations of the patient cohort

\begin{tabular}{lc}
\hline Clinical manifestation & No. of cases \\
\hline Aural fullness & 112 \\
Tinnitus & 20 \\
Temporomandibular joint pain while chewing & 39 \\
Clicking or crepitus of temporomandibular joint & 43 \\
Restricted mouth opening $(<40 \mathrm{~mm})$ & 15 \\
Unilateral mastication & 87 \\
Pain or discomfort in temporomandibular joint & 95 \\
Mandibular movement with deflection to the affected & 27 \\
$\quad$ side on opening & \\
Temporomandibular joint habitual dislocation & 5 \\
Temporomandibular joint injury & 3 \\
\hline
\end{tabular}

Table 2. Correlation between aural fullness and temporomandibular joint disorders of the patient cohort

\begin{tabular}{lcccc}
\hline RDC/TMD & \multicolumn{3}{c}{ Aural fullness } & Total \\
\cline { 2 - 4 } \multicolumn{1}{c}{ classification } & Grade 1 & Grade 2 & Grade 3 & \\
\hline Group I & 5 & 53 & 10 & $68(60.7)$ \\
Group II & 3 & 29 & 7 & $39(34.8)$ \\
Group III & 1 & 2 & 2 & $5(4.5)$ \\
Total & 9 & 84 & 19 & 112 \\
\hline
\end{tabular}

RDC/TMD, Research Diagnostic Criteria for Temporomandibular Disorders.

to examine the temporomandibular joint of all the patients. Three patients were also examined by CT scan. Forty patients showed abnormalities including narrowing or widening of the joint space, extrusion of the articular disk of mandibular joint, roughing of the joint surface, or cortical bone loss and adhesion. Of the 72 patients that showed normal temporomandibular joint structure, 68 were categorized as group I disorders (muscle disorders) based on the RDC/TMD [9]. The remaining 4 patients were classified to group II disorders (disc displacements) based on their history of temporomandibular joint injury, together with other 35 patients (a total of 39). Five patients belong to group III disorders (arthralgia, osteoarthritis, and osteoarthrosis). A grading system was established to assess the severity of aural fullness. Correlation between aural fullness and TMD classification was shown in Table 2.

\section{Effectiveness of TMD treatment}

Patients were treated sequentially by physiotherapy, glucocorticoid or sodium hyaluronate injection, orthodontics, occlusal equilibration, etc. as described above. Treatment outcomes are summarized in Fig. 1. Overall, treatments aimed at improving TMD completely resolved aural fullness in 67 patients and significantly improved the symptom in 34 patients, with a combined effectiveness of $90.2 \%$ (101/112). Specifically,TMD treatment effectiveness for aural fullness was $94.1 \%$ (64/68) for group I disorders (muscle disorders), with the majority (58/68) responding to physiotherapy. The group II disorders (disc displacements) of $84.6 \%$ (33/39) and group III disorders (arthral-



Fig. 1. Temporomandibular joint disorders treatment results of aural fullness in the patient cohort. E, effective; I, ineffective.

gia, osteoarthritis, and osteoarthrosis) of $80 \%$ (4/5) showed significant improvement of aural fullness following TMD treatments. Among the various treatments, physiotherapy showed the highest overall effectiveness (80/132) and was especially effective (58/68) for patients having group I disorders. Patients who did not respond to TMD treatments include those displaying temporomandibular joint articular cavity structure change (4 cases), osteoarthrosis (1 case), occlusal equilibration failure (4 cases), and habitual temporomandibular joint dislocation (2 cases). Forty-five patients voluntarily participated in follow-up surveys 10-18 months posttreatments. The aural fullness relapsing rate is $10.3 \%(3 / 29)$ for group ITMD disorders, $26.7 \%(4 / 15)$ for group II disorders, and $0 \%$ (0/1) for group III disorders respectively (Table 3). 
Table 3. Clinical follow-up of 45 patients

\begin{tabular}{lcc}
\hline RDC/TMD classification & Follow-up case & Recurrence case \\
\hline Group I & 29 & 3 \\
Group II & 15 & 4 \\
Group III & 1 & 0 \\
Total & 45 & 7 \\
\hline
\end{tabular}

RDC/TMD, Research Diagnostic Criteria for Temporomandibular Disorders.

\section{DISCUSSION}

Aural fullness is a common aural complaint, the etiology of which is not clearly defined. Previous studies have suggested a link between aural fullness and somatosensory dysregulation [10]. However, many patients complaining of aural fullness cannot correctly define the origin of the symptom [11]. In otolaryngology practice, patients with aural fullness as the main or sole complaint could be misdiagnosed. This study enrolled 112 patients who presented for aural fullness to the Otolaryngology Department, PLA Army General Hospital, Beijing, China, between January 2010 and January 2015. Of the 112 patients, 90 had been diagnosed and treated for otitis media and 23 for sensorineural deafness. This is not surprising given that aural fullness is among the manifestations of these pathologies. However, none of patients showed improvement in aural fullness after receiving corresponding treatments, suggesting a non-otolaryngology origin of the symptom.

Further examination of these patients revealed signs of TMD, which involves the masticatory muscles, the temporomandibular joint and associated structures [2]. Common aural manifestations of TMD include restricted mouth opening, clicking or crepitus of temporomandibular joint, otalgia, tinnitus, hearing loss, etc. [37]. Based on the questionnaire results as well as data obtained from X-ray and/or CT scan of the temporomandibular joint, patients were diagnosed and further categorized according to the RDC/TMD [9]. The use of X-ray and CT scan in this study helps to reveal potential pathological changes in temporomandibular joint and associated structures. Interestingly, TMD patients with aural symptoms were reported to often have changes in middle ear impedance as shown by multiple frequency tympanometry [8]. However, all of the 112 patients enrolled in this study showed type A tympanometry, indicative of normal middle ear immittance. Further studies need to determine the relationship between TMD, middle ear immittance and aural fullness.

Patients were subsequently treated for TMD based on the disease category and responsiveness to therapy. Strikingly, $90.2 \%$ of the patients showed disappearance of or significant improvement in aural fullness, suggesting TMD as a cause of the aural fullness. TMD treatments are especially effective $(94.1 \%)$ for improving aural fullness experienced in group ITMD (muscle disorders) patients. Among the different treatments, physiotherapy which includes heat therapy, acupuncture and massage is the most effective. To the author's knowledge, there has been only one case report showing that ear fullness was successfully managed as a temporomandibular disorder [12]. The present survey represents the first to show with a large patient cohort that TMD treatment could successfully cure/improve aural fullness. Interestingly, TMD treatments are often ineffective of improving aural fullness in patients showing temporomandibular joint structural changes. It is worth noting that one limitation of this retrospective study is the lack of untreated control group. Considering the possible self-resolution of aural fullness in the long term, we cannot conclude on the exact percentage of patients in which TMD drives aural fullness. However, our data clearly suggest a link between aural fullness and TMD. It is not clear how TMD causes aural fullness, although it has been proposed that TMD patients with a clenching habit might have dysfunctional masticatory muscle and tensor tympani muscle, leading to aural fullness [12].

Based on the study, a diagnosis/treatment protocol could be proposed for patients with chief aural fullness complaint. Aural fullness arisen from otologic causes can usually be diagnosed by a holistic review of one's medical history, symptoms, and results of pure tone audiometry, acoustic immittance screen, X-ray and/ or CT scan. For example, Ménière's disease-induced aural fullness is often associated with fluctuations tinnitus, dizziness and nausea. Earwax embolism can be seen by blocked external auditory canal. Otitis media can be detected by pure tone audiometry, acoustic immittance screen, X-ray and/or CT scan. If the patient has normal external auditory canal, eardrum and mastoid process, and shows type A tympanogram, TMD could underlie the aural fullness and should be further evaluated by commonly associated manifestations such as restricted mouth opening, clicking or crepitus of temporomandibular joint, etc.

In summary, this study establishes TMD as a potential cause of aural fullness and shows that TMD treatments are successful against aural fullness. Patients with aural fullness as the main or sole complaint should be examined for TMD. Once TMD is confirmed, physiotherapy, intra-articular injection of glucocorticoid and sodium hyaluronate, orthodontics and occlusal equilibration could be given in a sequential manner according to individual's TMD category and responsiveness to therapy.

\section{CONFLICT OF INTEREST}

No potential conflict of interest relevant to this article was reported.

\section{REFERENCES}

1. Greene CS. Managing the care of patients with temporomandibular disorders: a new guideline for care. J Am Dent Assoc. 2010 Sep;141 
(9):1086-8.

2. Durham J, Newton-John TR, Zakrzewska JM. Temporomandibular disorders. BMJ. 2015 Mar;350:h1154.

3. Akhter R, Morita M, Ekuni D, Hassan NM, Furuta M, Yamanaka R, et al. Self-reported aural symptoms, headache and temporomandibular disorders in Japanese young adults. BMC Musculoskelet Disord. 2013 Feb;14:58.

4. Buergers R, Kleinjung T, Behr M,VielsmeierV. Is there a link between tinnitus and temporomandibular disorders? J Prosthet Dent. 2014 Mar;111(3):222-7.

5. Cox KW. Temporomandibular disorder and new aural symptoms. Arch Otolaryngol Head Neck Surg. 2008 Apr;134(4):389-93.

6. Kitsoulis P, Marini A, Iliou K, Galani V, Zimpis A, Kanavaros P, et al. Signs and symptoms of temporomandibular joint disorders related to the degree of mouth opening and hearing loss. BMC Ear Nose Throat Disord. 2011 May;11:5.

7. Tuz HH, Onder EM, Kisnisci RS. Prevalence of otologic complaints in patients with temporomandibular disorder. Am J Orthod Dento- facial Orthop. 2003 Jun;123(6):620-3.

8. Riga M, Xenellis J, Peraki E, Ferekidou E, Korres S. Aural symptoms in patients with temporomandibular joint disorders: multiple frequency tympanometry provides objective evidence of changes in middle ear impedance. Otol Neurotol. 2010 Dec;31(9):1359-64.

9. Dworkin SF, LeResche L. Research diagnostic criteria for temporomandibular disorders: review, criteria, examinations and specifications, critique. J Craniomandib Disord. 1992 Fall;6(4):301-55.

10. SakataT, Higuchi H, UenoT, Nakagawa T. Modulation of somatosensory abilities and the feeling of ear fullness in patients with acute sensorineural hearing loss. Auris Nasus Larynx. 2012 Jun;39(3):2659.

11. Park MS, Lee HY, Kang HM, Ryu EW, Lee SK, Yeo SG. Clinical manifestations of aural fullness. Yonsei Med J. 2012 Sep;53(5):985-91.

12. Dalla-Bona D, Shackleton T, Clark G, Ram S. Unilateral ear fullness and temporary hearing loss diagnosed and successfully managed as a temporomandibular disorder: a case report. J Am Dent Assoc. 2015 Mar;146(3):192-4. 